๑

\title{
Mental and somatic symptoms related to suicidal ideation in patients visiting a psychosomatic clinic in Japan
}

This article was published in the following Dove Press journal:

International Journal of General Medicine

19 August 2009

Number of times this article has been viewed

\author{
Kouichi Yoshimasu' \\ Tetsuya Kondo2,4 \\ Shoji Tokunaga ${ }^{3}$ \\ Yoshio Kanemitsu ${ }^{2}$ \\ Hideyo Sugahara ${ }^{2}$ \\ Mariko Akamine ${ }^{2}$ \\ Kanichiro Fujisawa ${ }^{2}$ \\ Kazuhisa Miyashita' \\ Chiharu Kubo \\ 'Department of Hygiene, School \\ of Medicine, Wakayama Medical \\ University, Wakayama, Japan; \\ ${ }^{2}$ Department of Psychosomatic \\ Medicine, Graduate School of Medical \\ Sciences, Kyushu University, Fukuoka, \\ Japan; ${ }^{3}$ Department of Medical \\ Informatics, Kyushu University \\ Hospital, Fukuoka, Japan; ${ }^{4}$ Department \\ of Acupuncture and Moxibustion, \\ Kansai University of Health Sciences, \\ Osaka, Japan
}

Correspondence: Kouichi Yoshimasu Department of Hygiene, School of Medicine, Wakayama Medical University, 8-I-I Kimiidera, Wakayama 64I-00I2, Japan

Tel $+8 \mid 73$ 44I 0646

Fax $+8|7344| 0646$

Email kyoshi@wakayama-med.ac.jp

\begin{abstract}
Patients with suicidal ideation (SI) have various mental or somatic symptoms. A questionnaire-based interview elicited details concerning mental and somatic symptoms in patients visiting a psychosomatic clinic in Japan. Univariate logistic regression analyses followed by multiple regression models using a stepwise method were selected for identifying the candidate symptoms. Overall, symptoms related to depression were associated with SI in both sexes. Although women showed more various somatic symptoms associated with SI than men, many of those associations were diminished once severity of the depression was controlled. The current results suggest that a variety of self-reported symptoms, mainly related to depression, might reveal suicidal risk in outpatients with an urban hospital clinical setting. Keywords: suicidal ideation, psychosomatic clinic, subjective symptoms
\end{abstract}

\section{Introduction}

Recent figures show that more than 30,000 people commit suicide each year in Japan. Several epidemiological studies have indicated personal and social risk factors for suicide such as depression, severe anxiety, substance abuse, poor interpersonal relationships including social isolation, inability to maintain a job, anhedonia, somatic diseases, financial problems, and personal or familial history of suicide. ${ }^{1-7}$

Okuma $^{8}$ indicated the major causes for suicide as follows: 1) somatic or mental suffering caused by disease, social or familial conflict, 2) loss of something to live for, 3) psychosis, 4) emotional reaction, 5) overwork, 6) psychogenic reaction, 7) hostility, 8) escape from reality, and 9) borderline personality disorder. Especially, strategies for dealing with depression are regarded as a matter of high priority for preventing suicide in Japan..$^{5-7}$

In general, patients with depression are likely to consult a general physician rather than a psychiatrist in Western countries. ${ }^{8}$ However, psychosomatic clinics are relatively common in Japan, especially since 1996 when it became legal to open such clinics, and patients with less serious mental disorders such as neurotic, stress-related, and somatoform disorders as well as mild depression and stress-related physical disorders have tended to visit such clinics. Physicians with expertise of psychosomatic medicine treat these patients. If patients had more serious mental symptoms such as hallucination or delusion, they were sent to the department of neuropsychiatry.

Indeed, nearly half of new patients who visited psychosomatic clinics were reported to be suffering from depressive symptoms. ${ }^{9}$ Furthermore, nearly $30 \%$ of patients who visited a psychosomatic clinic in a university hospital were shown to suffer from mild 
depression, and more than $90 \%$ of their chief complaints were found to be somatic symptoms. ${ }^{10}$ Another study reported that $75 \%$ of the chief complaints were somatic symptoms in patients diagnosed with depression who visited a psychosomatic clinic in a university hospital. ${ }^{11}$ From this point of view, patients who visit a psychosomatic clinic are considered to be those with various mental and somatic disorders, in other words, those with equivocal symptoms. In addition, somatic symptoms were also shown to be associated with suicidal ideation (SI) in patients visiting a psychosomatic clinic, ${ }^{12}$ and chronic pain has been also reported to be associated with an increased suicidal risk. ${ }^{13}$

However, there may be some differences between men and women in mental or somatic symptoms which predict suicidal acts. Indeed, in the analyses using a part of the present subjects diagnosed with a major depressive disorders according to the Diagnostic and Statistical Manual of Mental Disorders Fourth Edition (DSM-IV), ${ }^{14}$ high anxiety state assessed by the State-Trait Anxiety Inventory (STAI) was more strongly associated with SI in women, suggesting that anxiety-related somatic symptoms may also be related to SI. It was also reported that there was an association between somatization and SI among 40 patients with dissociative disorders, among whom $80 \%$ was female. ${ }^{15}$ In addition, women with somatization disorder outnumber men five to 20 times. ${ }^{16}$ Thus, women are generally more likely to transform mental problems into somatic ones.

In the present study, we investigated the mental and somatic symptoms related to SI in Japanese patients who visited the department of psychosomatic medicine in a university hospital, focusing on the sex differences of these symptoms.

\section{Methods}

\section{Subjects}

There were 914 new patients (328 men and 586 women) who had check-ups at the Department of Psychosomatic Medicine in a university hospital in Kyushu area, Japan during the period from June 2000 to March 2001. Because this hospital is one of only several university hospitals that have a psychosomatic clinic in Japan, the patients who visited the clinic could be considered to be representative of patients in other university hospitals. Although new patients were to be referrals, approximately half of them were walk-in patients at our tertiary medical care center.

Patients with missing SI data $(n=94)$ were excluded. Thus, 304 men and 516 women remained in the analysis. However, the number of the subjects in each regression model varied because of missing values in the relevant factors. The correct sample sizes are shown in the corresponding tables (Tables 1-5).

\section{Assessment of psychosocial and somatic symptoms}

A questionnaire-based interview elicited details concerning psychological factors and somatic symptoms. Data were collected in the present study as a part of the routine medical examinations, and the data were analyzed after the original data were transformed into anonymous form that could not be connected to the original medical records. All of the participant patients gave written informed consent for the relevant medical examinations. The protocol of the present survey was approved by the Research Ethics Committee of Graduate School of Medical Sciences, Kyushu University.

The patients were requested to answer questions based on the original medical examination and several psychological tests, and to identify their three most painful symptoms on a check sheet. We confirmed the presence of SI and related psychosocial and somatic factors by the Kyudai Medical Index (KMI), Kyushu University's original medical index. It was developed as a modification of the Cornell Medical IndexHealth Questionnaire for rapid screening. ${ }^{17}$ This measure consists of the following three major sections: anamnesis and family history, subjective mental symptoms, and subjective somatic symptoms. It was reported that this measure could distinguish healthy controls from patients with neurotic disorders and those with psychosomatic disorders. ${ }^{17}$

The patients were requested to choose dichotomous answers (yes/no), and to reveal their real intentions with

Table I Frequencies of suicidal ideation with and without mental disorders according to the sex

\begin{tabular}{|c|c|c|c|c|c|c|}
\hline \multirow[t]{3}{*}{ Mental disorders* } & \multicolumn{6}{|c|}{ Frequencies of SI (\%) } \\
\hline & \multicolumn{3}{|l|}{ Men } & \multicolumn{3}{|c|}{ Women } \\
\hline & DP & DN & $P$ & DP & DN & $P$ \\
\hline MDD & 55.3 & 21.4 & $<0.0001$ & 55.8 & 23.8 & $<0.0001$ \\
\hline $\mathrm{ED} * *$ & 42.9 & 33.5 & 0.69 & 59.7 & 33.5 & $<0.0001$ \\
\hline$A D$ & 34.6 & 33.5 & 0.88 & 27.6 & 39.4 & 0.033 \\
\hline SFD & 39.1 & 33.2 & 0.57 & 34.5 & 37.7 & 0.73 \\
\hline
\end{tabular}

Notes: *Patients whose diagnoses were unconfirmed were excluded. ${ }^{*}$ Fisher's exact test was used for men.

Abbreviations: SI, suicidal ideation; DP, disease positive; DN, disease negative; MDD, major depressive disorder, $\mathrm{n}=3 \mathrm{I}$ I for positive and 498 for negative; ED, eating disorder, $\mathrm{n}=74$ for positive and 627 for negative; $A D$, anxiety disorder, $\mathrm{n}=142$ for positive and 559 for negative; SFD, somatoform disorder, $n=52$ for positive and 649 for negative; SI, suicidal ideation. 
Table 2 Numbers and frequencies of mental disorders according to suicidal ideation in men and women

\begin{tabular}{|c|c|c|c|c|c|c|}
\hline \multirow[t]{3}{*}{ Mental disorders* } & \multicolumn{6}{|c|}{ Numbers (\%) of mental disorders } \\
\hline & \multicolumn{3}{|l|}{ SI (+) } & \multicolumn{3}{|l|}{ SI (-) } \\
\hline & Men & Women & $P$ & Men & Women & $P$ \\
\hline MDD & $57(57.6)$ & $116(61.7)$ & 0.50 & $46(23.0)$ & $92(28.6)$ & 0.16 \\
\hline ED & $3(3.4)$ & $40(24.2)$ & $<0.0001$ & $4(2.3)$ & $27(9.8)$ & 0.0023 \\
\hline$A D$ & $19(21.6)$ & $24(14.6)$ & 0.16 & $36(20.8)$ & $63(22.9)$ & 0.60 \\
\hline SFD & $9(10.2)$ & $10(6.1)$ & 0.23 & $14(8.1)$ & $19(6.9)$ & 0.64 \\
\hline
\end{tabular}

Note: *Patients whose diagnoses were unconfirmed were excluded.

Abbreviations: SI, suicidal ideation; MDD, major depressive disorder, $\mathrm{n}=299$ for men and 510 for women; ED, eating disorder, AD, anxiety disorder; SFD, somatoform disorder, $\mathrm{n}=26 \mathrm{I}$ for men and 440 for women.

a guarantee of confidentiality. They were also requested to give intuitive responses, because serious thinking may confuse them. The question regarding SI was included in KMI asked "Do you often think you want to die?" If the patients answered as "yes" to this question, they were regarded as having SI. McNemar's test revealed no significant differences between this question and the SI-related item included in Zung's self-rating depression scale (SDS) in the subgroup of the present subjects for analyzing relation of sleep disorders and SI. ${ }^{18}$ However, only the KMI question was used in the present study for avoiding missing data arising due to inconsistency between these two questions.

\section{Diagnoses of mental disorders}

First, a physician in charge of outpatients interviewed all the patients and made provisional diagnoses. The patients

Table 3 Relation of mental symptoms to suicidal ideation: Results of logistic procedure using stepwise method

\begin{tabular}{|c|c|c|c|c|c|c|}
\hline \multirow[t]{2}{*}{ Variable } & \multicolumn{3}{|c|}{ Men $(n=195)$} & \multicolumn{3}{|c|}{ Women $(n=262)$} \\
\hline & $\mathbf{O R}^{*}$ & $95 \% \mathrm{Cl}$ & $P$ & $\overline{\text { OR* }^{*}}$ & $95 \% \mathrm{Cl}$ & $P$ \\
\hline Feel agitated & 4.28 & $1.07-17.19$ & $0.04 I$ & 2.06 & $0.73-5.81$ & 0.17 \\
\hline Lack of self-confidence & 19.95 & $2.16-184.57$ & 0.0084 & - & - & \\
\hline Unpleasantness at home and work & 7.31 & $1.97-27.14$ & 0.0030 & 2.65 & $1.22-5.79$ & 0.014 \\
\hline Often give up when planning things & 2.35 & $0.78-7.04$ & 0.13 & 1.71 & $0.83-3.51$ & 0.14 \\
\hline Angry when things don't go my way & - & - & & 2.35 & $0.91-6.05$ & 0.078 \\
\hline Uncomfortable with people & - & - & & 0.33 & $0.14-0.78$ & 0.012 \\
\hline Exaggerate things when talking & 0.15 & $0.04-0.55$ & 0.0038 & - & - & \\
\hline Worried, not much peace lately & 0.23 & $0.04-1.45$ & 0.12 & - & - & \\
\hline Nervous about what others say & 0.30 & $0.09-1.01$ & 0.052 & - & - & \\
\hline Sweat or tremble when stressed out & 0.23 & $0.07-0.80$ & 0.021 & 1.78 & $0.87-3.63$ & 0.11 \\
\hline Feel hopeless & - & - & & 0.24 & $0.09-0.60$ & 0.0022 \\
\hline Hard to please & 2.57 & $0.87-7.59$ & 0.088 & - & - & \\
\hline Something wrong with my mind or body & 3.44 & $0.56-21.01$ & 0.18 & 3.11 & $1.12-8.64$ & 0.030 \\
\hline Afraid of a certain disease & - & - & & 2.00 & $0.91-4.39$ & 0.085 \\
\hline Sickness depends on my mood & 0.38 & $0.11-1.30$ & 0.12 & - & - & \\
\hline Can't get rid of obsessive thoughts & 4.39 & $1.15-16.84$ & 0.031 & 2.40 & $1.10-5.22$ & 0.028 \\
\hline Depressive mood & 5.84 & $1.90-17.98$ & 0.0021 & 4.31 & $1.96-9.46$ & 0.0003 \\
\hline Afraid to mix in & - & - & & 2.12 & $1.03-4.33$ & $0.04 I$ \\
\hline Self-reproach & - & - & & 1.82 & $0.82-4.04$ & 0.14 \\
\hline Derealization & - & - & & 2.26 & $1.05-4.86$ & 0.037 \\
\hline Feelings of persecution & II.4I & $2.93-44.47$ & 0.0005 & - & - & \\
\hline Depersonalization & $5.7 I$ & $1.57-20.82$ & 0.0083 & - & - & \\
\hline
\end{tabular}

Notes: *Age was included in the model. Bold numbers indicate statistically significant associations. The above variables were selected through stepwise procedure in each model according to sex.

Abbreviations: $\mathrm{Cl}$, confidence interval; OR, odds ratio. 
Table 4 Relation of somatic symptoms to suicidal ideation: Results of logistic procedure using stepwise method

\begin{tabular}{|c|c|c|c|c|c|c|}
\hline \multirow[t]{2}{*}{ Variable } & \multicolumn{3}{|c|}{ Men $(n=177)$} & \multicolumn{3}{|c|}{ Women $(n=216)$} \\
\hline & OR* & $95 \% \mathrm{Cl}$ & $P$ & OR* & $95 \% \mathrm{Cl}$ & $P$ \\
\hline General fatigue & 4.11 & $1.04-16.29$ & 0.045 & - & - & \\
\hline Feeling of listlessness & 0.30 & $0.08-1.07$ & 0.062 & - & - & \\
\hline Lack of persistence & 2.29 & $0.7 \mathrm{I}-7.35$ & 0.16 & 3.54 & $1.56-8.05$ & 0.0026 \\
\hline Chest pain & - & - & & 2.63 & $1.04-6.65$ & 0.040 \\
\hline Heavy feeling in chest & 0.20 & $0.05-0.77$ & 0.020 & - & - & \\
\hline Difficulty breathing & 9.32 & $2.60-33.39$ & 0.0006 & - & - & \\
\hline Edema & - & - & & 4.49 & $1.82-11.07$ & 0.0011 \\
\hline Tinnitus & - & - & & 3.80 & $1.48-9.75$ & 0.0055 \\
\hline Headache & - & - & & 0.37 & $0.15-0.87$ & 0.023 \\
\hline Falling with loss of consciousness & 0.24 & $0.07-0.85$ & 0.026 & 0.26 & $0.10-0.70$ & 0.0077 \\
\hline Difficulty falling asleep & - & - & & 2.77 & $1.25-6.14$ & 0.012 \\
\hline Short sleep & - & - & & 1.96 & $0.86-4.45$ & 0.11 \\
\hline Frequent dream & - & - & & 3.26 & $1.19-8.88$ & 0.021 \\
\hline
\end{tabular}

Notes: *Age and the total score of self-rating depression scale were included in the model. Bold numbers indicate statistically significant associations. The above variables were selected through stepwise procedure in each model according to sex.

Abbreviations: $\mathrm{Cl}$, confidence interval; $\mathrm{OR}$, odds ratio.

were then assigned to individual physicians in charge of routine medical examinations, and given a complete checkup. These diagnoses were all conducted according to DSM-IV criteria.

As mental disorders commonly observed in psychosomatic clinic, the frequencies of major depressive disorder (MDD), eating disorders (ED), anxiety disorders (AD), and somatoform disorders (SFD) as well as their associations with SI were checked in the present survey. The severity of the depressive state of each patient was also evaluated by the Japanese version of the SDS.

\section{Statistical analysis}

Logistic regression analysis was used for univariate and multivariate analyses. The outcome variable was the SI

Table 5 High number of somatic symptoms in relation to sex and suicidal ideation $(n=412)^{*}$

\begin{tabular}{llll}
\hline $\begin{array}{l}\text { Combination } \\
\text { of sex and SI }\end{array}$ & $\begin{array}{l}\text { Age-adjusted } \\
\text { OR }\end{array}$ & $\mathbf{9 5 \%} \mathbf{C l}$ & $\mathbf{P}$ \\
\hline Men and SI (-) & I.00 & Ref & \\
Women and SI (-) & 1.34 & $0.82-2.17$ & 0.29 \\
Men and SI (+) & $\mathbf{4 . 4 8}$ & $\mathbf{2 . 2 1 - 9 . 0 6}$ & $<\mathbf{0 . 0 0 0 ~ I}$ \\
Women and SI (+) & $\mathbf{6 . 6 2}$ & $\mathbf{3 . 4 5 - 1 2 . 7 0}$ & $<\mathbf{0 . 0 0 0 I}$ \\
\hline
\end{tabular}

Notes: Bold numbers indicate statistically significant associations. *Number of somatic symptoms of 14/45 or more was defined as a high number of somatic symptoms. Only patients without any missing values of the 45 somatic symptoms in the Kyudai Medical Index were included in the analysis.

Abbreviations: $\mathrm{Cl}$, confidence interval; $\mathrm{OR}$, odds ratio; $\mathrm{SI}$ suicidal ideation. assessed by KMI, and all the other items included in KMI regarding mental and somatic symptoms were used as explanatory variables. The patients without these symptoms were regarded as the referent group and were compared with those having positive symptoms.

The analytic procedure was conducted according to the following two steps. First, univariate logistic regression analysis was performed separately by sex. Next, multivariate logistic regression analysis according to sex was performed using the stepwise method for selecting variables. The explanatory variables included in the multivariate logistic regression models were those which were statistically significantly associated with SI in the univariate analysis. These variables were classified into two categories: subjective mental symptoms and subjective somatic symptoms. Age was included in each model and the total score of SDS was additionally included in the analysis of somatic symptoms as continuous variable, because the severity of depression is considered to be an important confounding factor between SI and somatic symptoms. All the explanatory variables except age were divided into two covariate categories (yes/no), and the adjusted odds ratio (OR) and $95 \%$ confidence interval (CI) were obtained from the corresponding logistic regression coefficients and their standard errors. Age was divided into three categories $(<26,26-50$, and $51+)$, because the suicide rate increases in the twenties and fifties age brackets. ${ }^{19}$ For stepwise selections, the significance level for both entry and removal was set at 0.20 . 
To evaluate the interactive effect between sex and SI on somatic symptoms, the total number of somatic symptoms except two items only for women were accumulated (coded as yes $=1$, no $=0$ ) and divided into two categories at median value. Both sex and SI were included in the logistic regression model using categorized somatic symptoms as a dependent variable.

P-values (two-sided) less than 0.05 were considered statistically significant. All computations were performed using the SAS software package (version 9.1.3; SAS Institute, Inc., Cary, NC, USA).

\section{Results}

The mean ages of men $(n=304)$ and women $(n=516)$ were 36.9 years (SD 16.4) and 37.5 years (SD 17.8), respectively. The frequencies of SI in men and women were $32.6 \%$ and $36.3 \%$, respectively. Approximately half of both men and women were in their twenties and thirties.

Table 1 shows the frequency of SI according to mental disorders. SI was more frequent in patients with MDD in both men and women $(P<0.0001)$. The frequency of SI did not statistically differ between patients with and without the other disorders, except that SI was more frequent in female patients without $\operatorname{AD}(P=0.033)$ and with $\operatorname{ED}(P<0.0001)$.

Table 2 shows differences in the frequencies of the above four mental disorders between men and women according to the presence of SI. In patients with SI, almost $60 \%$ of both men and women were diagnosed as MDD. ED was statistically significantly more frequent in women than in men regardless of SI. Frequencies of the other three disorders were not significantly different between both sexes in patients with SI as well as those without SI. Thus, in the present study, sex differences of symptoms associated with SI, which were caused by disease bias, were considered to be negligible.

In univariate analysis, 43 items of 49 subjective mental symptoms and 26 items of 45 subjective somatic symptoms were statistically significantly associated with an increased or decreased risk of SI in men, while 39 mental symptoms and 38 somatic symptoms were associated in women. In women, two questions regarding menstruation were added to those in men as somatic symptoms. Overall, women showed more various somatic symptoms positively or negatively associated with SI than men despite the fact that the numbers of mental symptoms associated with SI between men and women were almost equal.

As for frequencies of mental or somatic symptoms according to sex, seven mental symptoms (nightmare, being tied to surprising event, decline of motivation, fear of bad news, hopelessness, fear of a specific object, loneliness) and eight somatic symptoms (infirmity, palpitation, edema, sensitivity to cold, nausea, diarrhea or constipation, dermatitis, numbness) were statistically significantly more frequent in women, whereas two mental symptoms (uncomfortableness with people, being hard to please) and two somatic symptoms regarding sexual problems were more frequent in men. The frequencies of the other somatic and mental symptoms were not statistically significantly different between men and women.

The results from multiple logistic regression analysis by stepwise method were shown in Tables 3 and 4. Subjective mental symptoms associated with SI in men and women were presented in Table 3. Unpleasant experiences at home or a work, obsessive ideas, and depressive moods were statistically significantly associated with an increased risk of SI in both men and women. Agitation, loss of self-confidence, ideation of persecution, and depersonalization were statistically significantly associated with SI in men, but not in women. On the other hand, feelings that one's mental or physical condition is poor, agoraphobia, and derealization were significantly associated with SI in women. Exaggerating things when talking and poor physical conditions when under mental stress were significantly associated with a decreased risk of SI in men, whereas uncomfortable feelings around people or hopelessness were negatively associated with SI in women.

Table 4 shows subjective somatic symptoms related to SI according to sex. Only common symptom tended to be associated with an increased risk of SI in both men and women was lack of persistence. Interestingly, falling with loss of consciousness was statistically significantly associated with a decreased risk of SI in both sexes. General fatigue and difficulty breathing were specifically associated with an increased risk of SI in men, while chest pain, edema of the arms, legs or face, tinnitus, and difficulty in falling asleep as well as frequent dream were statistically significantly associated with it in women. Heavy feeling in chest and headache were associated with a decreased risk of SI in men and women, respectively.

Table 5 shows the combined influence of sex and SI on the number of somatic symptoms. Although being women and having SI were most strongly associated with a higher number of somatic symptoms, the interaction between sex and SI was far from statistical significance (likelihood ratio test, $P=0.83$ ).

\section{Discussion}

The present study revealed the mental and somatic symptoms associated with SI according to gender. As a whole, mental 
symptoms directly related to depression were shown to be associated with SI in both men and women. Although some self-reported mental symptoms associated with SI differed in men and women, those symptoms were similar in that they could be also regarded as some kinds of depressive symptoms and threats to one's identity.

Likewise, the observed somatic symptoms associated with SI in both genders were those related to depression. Indeed, many of them were consistent with those reported as frequent somatic symptoms observed in depression. ${ }^{8}$ Women showed more various symptoms associated with SI than men in univariate analysis. However, many of such associations were diminished, and the gender differences have been brought into sharp relief when the severity of depressive state was controlled. Although women with SI showed most strong association with an increased risk of high number of somatic symptoms, no substantial interaction was observed between gender and SI on the number of somatic symptoms.

In the present study, $311(37.9 \%)$ of the 820 patients were diagnosed with MDD. However, the severity of depression could be considered relatively mild because the Department of Psychosomatic Medicine is independent of the Neuropsychiatry Department in our hospital. Thus, patients with a severer degree of depression are more likely to undergo a check-up in the latter department. This was also supported by the fact that the mean score of SDS in the present patients diagnosed with MDD was slightly more than 50 points. ${ }^{14}$ An increasing number of such mild depression cases has been noted in the primary care medical setting. ${ }^{10}$ The observed depression-related mental and somatic symptoms associated with SI in the present subjects suggested that the screening and treatment of depression might be a matter of highest priority in the strategy of suicide prevention, ${ }^{5}$ even in a psychosomatic clinic with mild depressive patients.

Some epidemiological studies suggested that a lack of social support was a great risk of suicide in elderly Japanese people. ${ }^{6,20}$ Although the mean age of the present subjects was 30-39 years, poor relationships at home or work were associated with SI in both men and women. This effect remained even after controlling depressive moods. Foreign case-control studies with subjects of similar age construction with the present one indicated that poor social or family support were associated with suicidal risk. ${ }^{21,22}$

The present study also revealed that women tended to show more various somatic symptoms associated with SI than men. However, diminishing associations between somatic symptoms and SI after adjusting for SDS score suggested that a large extent of these associations might be explained by the depressive state of women visiting the psychosomatic clinic. Although the previous study did not find any material association between chest pain and $\mathrm{SI},{ }^{12}$ the current study revealed a strong association between them. Most patients who present to the emergency department of hospital for chest pain do not have a cardiac disease, but $30 \%$ of them suffer from panic disorder which was reported to be a risk factor of SI. ${ }^{23,24}$ In addition, women are two to three times more likely to be affected with panic disorder than are men. ${ }^{25}$ As mentioned earlier, a high anxiety state was more strongly associated with SI in women with MDD in the present subjects by multivariate analysis. ${ }^{14}$ Thus, chest pain, which is related to anxiety, may be a critical sign of SI in women. On the other hand, Nakao and colleagues ${ }^{12}$ did not find any significant association between anxiety and SI, in which both men and women were combined. This may be one explanation for the inconsistency between the current study and that by Nakao and colleagues. ${ }^{12}$

Although the high correlation was observed between diagnosis of AD and state anxiety assessed by STAI in both previous $^{14}$ and current study, there was a great discrepancy in the relation of this anxiety assessment to SI in women. The comorbidity of MDD was significantly more frequent in patients without $\mathrm{AD}$ than those with $\mathrm{AD}$ especially in women $(P=0.021)$. This may be one explanation why SI was more frequent in patients without $\mathrm{AD}$. Because the present subjects were patients visiting a psychosomatic clinic, patients without $\mathrm{AD}$ tended to have the other diagnoses such as MDD. On the other hand, high score of state anxiety revealed the high anxiety level at the time of having medical examinations. It is plausible that the patients with existing high anxiety level may have feeling like SI.

Interestingly, exaggerating things when talking and poor physical conditions when under heavy mental stress were inversely associated with a risk of SI in men whereas uncomfortable feelings with people was negatively associated with SI in women. These symptoms may be, in a sense, defensive reactions to mental or physical strain. In other words, they may be some kind of "flight into illness" which is considered to be a major psychological mechanism related to the onset of dissociative disorders (hysteria). This defense mechanism against mental strain or intrapsychic conflicts may tentatively avoid intolerable anxiety, and defuse the risk of triggering SI. Falling with loss of consciousness in daily lives, heavy feelings in the chest in men, or headache in women might 
be also connected with this defense mechanism. Indeed, dissociative disorders are characterized in part by personality with exaggerated expression. ${ }^{8}$

The fact that feeling hopeless was inversely associated with SI in women was not ordinary and difficult to interpret. In the present study, hopelessness was assessed by the following one question 'Do you worry yourself needlessly about the future?' Although patients with various degrees of worrying states may be contained in those with a 'yes' response to this question, a moderate degree of worrying may also reflect a defensive psychological mechanism against SI. Simultaneously, this item may also reflect anxiety disorder as resulting in negative association with SI.

Generalization of the present results is the major limitation included in the present study. Needless to say, it is difficult to apply findings derived from clinical sample to a general population. In this regard, power to detect effects of the symptoms on SI might be stronger for women because of their larger sample size than men. Female/male ratio of new outpatients amounted through three years in other psychosomatic clinic was reported to be $1.3,{ }^{9}$ which is similar to that of the present study remaining in the multivariate analyses. Thus, women having both neurotic tendency and somatic symptoms might be overrepresented in Japanese psychosomatic clinic. However, the aims of the present study are describing plain clinical characteristics of patients visiting psychosomatic clinic as well as establishing the key contributing elements that would aid in discovering suicidal signs of these patients rather than extrapolation of the results into general population. In addition to this referral bias, the biases caused by many missing values should not be ignored. Despite these study limitations, the present findings can provide valuable information to clinicians at primary care institutions for noticing suicidal signs of patients.

\section{Conclusions}

The present cross-sectional study with a clinic-based sample demonstrated that mental and somatic symptoms generally related to depression were associated with SI in both men and women. Although women tended to have more various somatic symptoms associated with SI than men, many of them might be explained by the neurotic tendency of women visiting a psychosomatic clinic. These results provide additional evidence that treatment for depression as well as detection of depression is a high priority for preventing suicide in patients visiting a psychosomatic clinic.

\section{Disclosures}

The authors report no conflicts of interest in this work.

\section{References}

1. Sechter D, Bonin B, Bertschy G, Vandel S, Bizouard P. Prediction of suicide risk. Encephale. 1991;17:361-364.

2. Hall RC, Platt DE, Hall RC. Suicide risk assessment: a review of risk factors for suicide in 100 patients who made severe suicide attempts. Evaluation of suicide risk in a time of managed care. Psychosomatics. 1999;40:18-27.

3. Aihara H, Iki M. An ecological study of the relations between the recent high suicide rates and economic and demographic factors in Japan. J Epidemiol. 2003;13:56-61.

4. Nishi N, Kurosawa M, Nohara M, et al. Knowledge of and attitude toward suicide and depression among Japanese in municipalities with high suicide rates. J Epidemiol. 2005;15:48-55.

5. Fushimi M, Sugawara J, Shimizu T. Suicide patterns and characteristics in Akita, Japan. Psychiatry Clin Neurosci. 2005;59:296-302.

6. Awata S, Seki T, Koizumi Y, et al. Factors associated with suicidal ideation in an elderly urban Japanese population: A community-based, cross-sectional study. Psychiatry Clin Neurosci. 2005;59:327-336.

7. Fujioka S, Abe S, Hiraiwa K. Lifetime social, psychological and physical background of suicides-research on the number of suicides during a year in Fukushima Prefecture. Seishin Shinkeigaku Zasshi. 2004;106:17-31. [Article in Japanese].

8. Okuma H. Modern Clinical Psychiatry. 11th ed. Tokyo, Japan: Kanahara Press; 2008. [Book in Japanese].

9. Miki O. The clinical feature of depression with psychosomatic medicine in primary care. Jpn J Psychosom Med. 2002;42:585-591. [Article in Japanese].

10. Kuboki T. The state of psychosomatic medicine in a university hospital. Seishinigaku. 2005;47:238-240. [Article in Japanese].

11. Sugahara H, Akamine M, Kondo T, et al. Somatic symptoms most often associated with depression in an urban hospital medical setting in Japan. Psychiatry Res. 2004;128:305-311.

12. Nakao M, Yamanaka G, Kuboki T. Suicidal ideation and somatic symptoms of patients with mind/body distress in a Japanese psychosomatic clinic. Suicide Life Threat Behav. 2002;32:80-90.

13. Tang NK, Crane C. Suicidality in chronic pain: a review of the prevalence, risk factors and psychological links. Psychol Med. 2006;36(5):575-586.

14. Yoshimasu K, Sugahara H, Tokunaga S, et al. Gender differences in psychiatric symptoms related to suicidal ideation in Japanese patients with depression. Psychiatry Clin Neurosci. 2006;60:563-569.

15. Öztürk E, Sar V. Somatization as a predictor of suicidal ideation in dissociative disorders. Psychiatry Clin Neurosci. 2008;62:662-668.

16. Kaplan HI, Sadock BJ. Somatoform disorders. In: Kaplan HI, Sadock BJ, editors. Synopsis of Psychiatry. 8th ed. Baltimore, MD: Lippincott Williams \& Wilkins; 1998. p. 632.

17. Matsuoka Y. KMI (Kyudai Medical Index). In: Kawano T, Suematsu H, Shinzato R, editors. Psychological Test for Psychosomatic Medicine. Tokyo: Asakura Shoten; 1990. pp. 45-49. [Article in Japanese].

18. Yoshimasu K, Sugahara H, Akamine M, et al. Sleep disorders and suicidal ideation in Japanese patients visiting a psychosomatic clinic in a university hospital. Sleep Biol Rhythms. 2006;4:137-143.

19. Health and Welfare Statistics Association. An outline of the cause of death. Journal of Health and Welfare Statistics. 2008;55:48-54. [Article in Japanese].

20. Oyama H, Watanabe N, Ono Y, et al. Community-based suicide prevention through group activity for the elderly successfully reduced the high suicide rate for females. Psychiatry Clin Neurosci. 2005;59:337-344.

21. Owens C, Booth N, Briscoe M, Lawrence C, Lloyd K. Suicide outside the care of mental health services. Crisis. 2003;24(3):113-121.

22. Kõlves K, Värnik A, Tooding LM, et al. The role of alcohol in suicide: a case-control psychological autopsy study. Psychol Med. 2006;36: 923-930. 
23. Fleet RP, Dupuis G, Marchand A, et al. Panic disorder in emergency department chest pain patients: prevalence, comorbidity, suicidal ideation, and physician recognition. Am J Med. 1996;101:371-380.

24. Fleet RP, Dupuis G., Kaczorowski J, et al. Suicidal ideation in emergency department chest pain patients: panic disorder a risk factor. Am J Emerg Med. 1997;15:345-349.
25. Kaplan HI, Sadock BJ. Panic disorder and agoraphobia. In: Kaplan HI, Sadock BJ, editors. Synopsis of Psychiatry. 8th ed. Baltimore, MD: Lippincott Williams \& Wilkins; 1998. p. 594.

\section{Publish your work in this journal}

The International Journal of General Medicine is an international, peer-reviewed open-access journal that focuses on general and internal medicine, pathogenesis, epidemiology, diagnosis, monitoring and treatment protocols. The journal is characterized by the rapid reporting of reviews, original research and clinical studies across all disease areas.
A key focus is the elucidation of disease processes and management protocols resulting in improved outcomes for the patient. The manuscript management system is completely online and includes a very quick and fair peer-review system. Visit http://www.dovepress.com/ testimonials.php to read real quotes from published authors.

Submit your manuscript here: http://www.dovepress.com/international-journal-of-general-medicine-journal 